EAGE

\title{
SSP02
}

\section{Applications of Common Reflection Angle} Migration

C. Tsingas* (Saudi Aramco), Z. Koren (Paradigm) \& M. Aloni (Paradigm) 
The structural interpretation of prestack depth migrated seismic data is based on stacking full offset flat depth gathers. Full offset stacking for complex subsurface environment can result in a lower signal to noise ratio than a partial angle stack of subsurface angle data. Common Reflection Angle Migration is a ray-based prestack depth migration that generates angle dependent reflectivity depth gathers providing the interpreter with a new way of viewing seismic data. Ray tracing is performed by shooting up-going rays for each subsurface image point with uniform emergence angles, thus multipathing arrivals are taken into account. Technically, this output-driven, upward-shooting approach enables one to compute migration operators that are more accurate than their Kirchhoff counterparts.

In this presentation we will illustrate a series of 2D and 3D Common Reflection Angle Migration synthetic and real data examples and compare the results obtained from a Kirchhoff prestack depth migration algorithm. In particular, we will illustrate the benefits of producing Angle Domain Common Image Gathers (ADCIG) to generate gathers that can be employed directly to an AVA impedance inversion workflow and to assist in the illumination and structural interpretation of highly complex regimes.

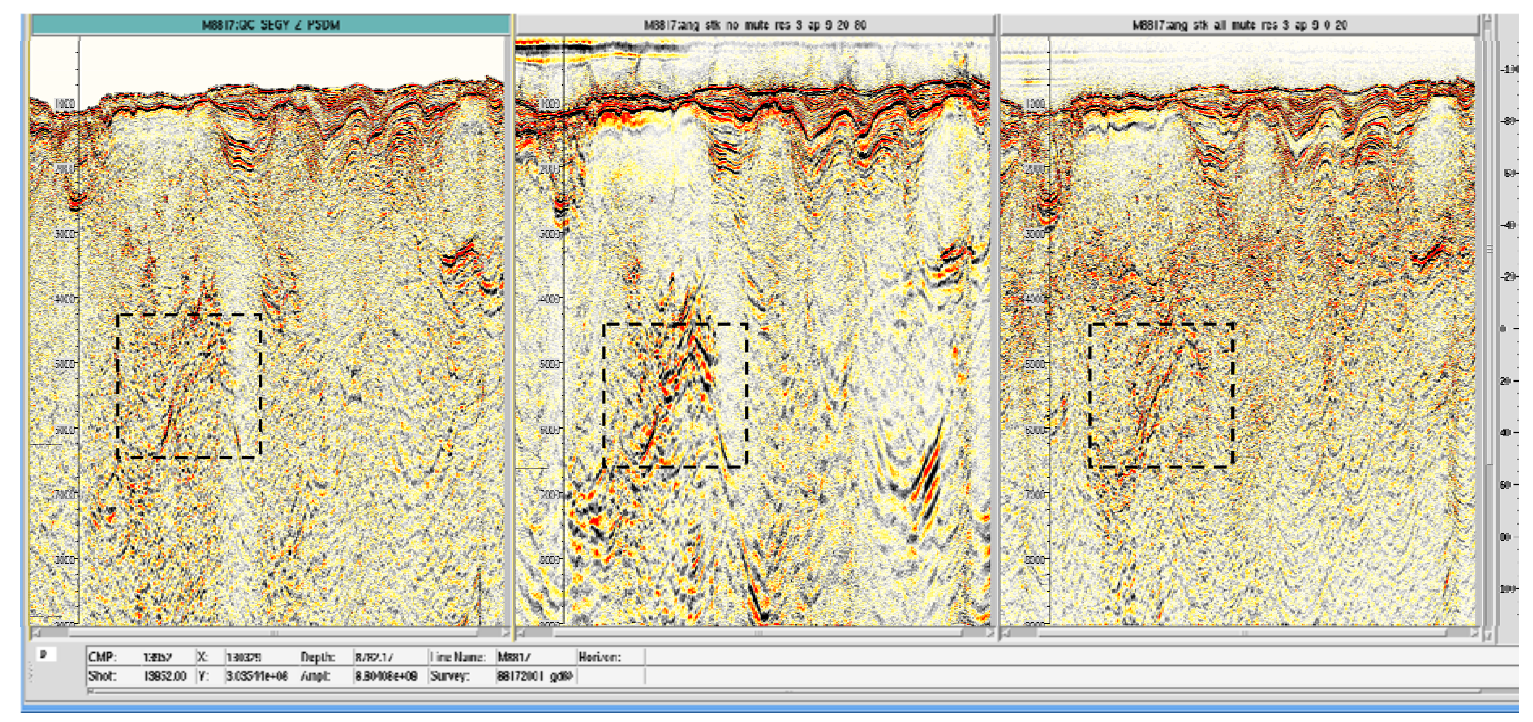

Figure 1 Left panel depicting the final Kirchhoff PSDM section (after multiple elimination applied in the time migrated domain, residual moveout, mute and stack). Middle panel depicts a 2D Common Reflection Angle Migration depth section produced by stacking all the ADCIGs with subsurface emergence angle ranging from 20 to 80 degrees (one pass of residual moveout and no mute was applied). Right panel is the 2D Common Reflection Angle Migration depth section produced by stacking all the ADCIGs with subsurface emergence angle ranging from 0 to 20 degrees. Note the main differences in the image sections highlighted by the boxes. 